\title{
Fast algorithms for short prime length fast biased polynomial transforms
}

\author{
J.-L. Wu
}

Indexing terms: Matrix algebra, Mathematical techniques

Abstract: In the paper, fast algorithms for three short prime length fast biased polynomial transforms are presented, based on the strategy of the minimum number of rotations. These algorithm are of practical use in digital image processing.

\section{Introduction}

Two dimensional (2D) digital convolution has many applications in digital signal processing, particularly in the processing of image, array and seismic signals. The conventional calculation for $2 \mathrm{D}$ digital convolution is evaluated by using discrete transform methods, such as fast Fourier transforms (FFTs) and number theoretic transforms (NTT), in column-row wise. These discrete transform methods suffer from disadvantages such as excessive multiplications in the FFT algorithms and limitations on convolution length and on dynamic range in the NTTs [1].

Polynomial transforms (PTs) were first developed by Nüssbaumer [2] to efficiently map 2D convolutions into 1D convolutions and polynomial products. Since all the operations of the PTs are defined in the rings of polynomials, the $2 \mathrm{D}$ convolution can be calculated by using ordinary arithmetic without multiplication, matrix transpose, dynamic range limitation and roundoff error [1]. In Nüssbaumer's approach the $Z^{N}-1$ polynomial ring is first factored into several irreducible cyclotomic poly nomials [3]. The cyclotomic polynomial subrings are then operated on by the PT. Finally, the results are combined using the Chinese remainder theorem (CRT) and column-row reordering processes. The CRT and reordering usually complicated the algorithm and the process.

Arambepola and Rayner [4] introduced a complex mapping to transform a circular convolution into a skewcircular one and vice versa. This enables the series of PTs to be replaced by a single $N$-point PT modulo $Z^{N}+1$. Owing to pre- and post multiplications by roots of unity, the improved algorithm actually introduced additional complex multiplications into the transform.

Recently, Wu and Pei [5] introduced a new PT algorithm which minimised these disadvantages. Instead of factoring out several irreducible cyclotomic polynomia subrings, the fast biased polynomial transform (FBPT) maps onto the $Z^{N}-1$ ring itself. This technique eliminates the complex mapping, CRT and column-row reordering processes. It is shown that polynomial convolution

Paper 7034E (C1, C2), first received 29th June 1988 and in revised form 28th September 1989

The author is with the Department of Computer Science and Information Engineering, National Taiwan University, Taipei 10764, Taiwan

IEE PROCEEDINGS, Vol. 137, Pt. E, No. 2, MARCH 1990 may be carried out without the need for multiplication. Since the $Z^{N}-1$ ring on which the FBPT is based is not in the form of cyclotomic polynomials, it will introduce biases in the final results. But these biases can be easily eliminated, especially when the transform length is a prime.

The FBPT has been applied for the computation of 2D digital convolution [6] and for the evaluation of 2D prime length discrete Fourier transforms (DFTs) [7]. For a composite length FBPT, an FFT-like algorithm was proposed in Reference 8; however, it is an open problem for developing fast algorithms to compute the prime length FBPTs.

The Winograd Fourier transform (WFT) is another well known approach for the computations of DFTs. Nevertheless, the WFT algorithms are designed for the minimum number of multiplications and, as a consequence, are not well suited in the computation of prime length FBPTs (because the underlying mathematical structure has completely changed).

More recently, Duhamel and Hollmann [9] presented some short prime length DFTs based on the strategy of the minimum number of rotations. In this paper, we extend the concept of 'minimum number of rotations' to the design of fast short prime length FBPTs. \section{Some fast algorithms for short prime length
FBPT}

In this Section, fast algorithms (with the minimum number of shifts) for $N$-point FBPT, where $N=3,5,7$ (practical lengths for 2D window function in image processing) are presented. The only allowed operations are polynomial additions and digit shifts (the multiplication by $Z$ or powers of $Z$ ); moreover, if necessary, each shifting operation is applied to a sum of previously computed terms.

The FBPT of a $2 \mathrm{D}$ input sequence $x(n, i), n, i=0, \ldots$ $N-1$, is defined as

$$
\tilde{X}(k, Z)=\sum_{n=0}^{N-1} X(n, Z) Z^{n k} \bmod \left(Z^{N}-1\right)
$$

where

$$
X(n, Z)=\sum_{i=0}^{N-1} x(n, i) Z^{i}
$$

$$
\text { and } k=0,1, \ldots, N-1 \text {. }
$$

For simplicity, we denote $\bar{X}(k, Z)$ and $X(n, Z)$ by $\bar{X}(k)$ and $X(n)$, respectively, and use $y(m), Y(m), H(m)$ as temporary storages for the intermediate results. Note that if the dummy variable $Z$ is replaced by the $N$ th root of unity, we can get the corresponding $N$-point minimal rotation DFTs given in Reference 9. 
(a) FBPT of length 3:

$Y(1)=Z(X(1)-X(2))$

$\bar{X}(0)=X(0)+X(1)+X(2)$

$\bar{X}(1)=X(0)-X(2)+Y(1)$

$\bar{X}(2)=X(0)-X(1)-Y(1)$

(b) FBPT of length 5 :

$$
\begin{aligned}
y(i) & =X(i)-X(4), i=1,2,3 \\
Y(1) & =Z^{3} y(1) \\
Y(2) & =Z^{2} y(2) \\
Y(3) & =Z y(3) \\
Y(4) & =Z^{3}(Y(1)+y(3)) \\
Y(5) & =Z^{2}(Y(2)+y(1)) \\
Y(6) & =Z^{4}(Y(2)+y(3)) \\
H(0) & =X(1)+X(2)+X(3)+X(4) \\
H(1) & =-X(4)+Y(4)+Y(2) \\
H(2) & =-X(4)+Y(5)+Y(3) \\
H(3) & =-X(4)+Y(6)+Y(1) \\
\bar{X}(i) & =X(0)+H(i), i=0,1,2,3 \\
\bar{X}(4) & =X(0)-H(0)-H(1)-H(2)-H(3)
\end{aligned}
$$

\begin{tabular}{|c|c|c|c|c|}
\hline \multirow[b]{2}{*}{$N$} & \multicolumn{2}{|c|}{$\begin{array}{l}\text { Proposed } \\
\text { algorithm }\end{array}$} & \multicolumn{2}{|c|}{$\begin{array}{c}\text { Direct } \\
\text { approach }\end{array}$} \\
\hline & $M(N)$ & $A(N)$ & $M(N)$ & $A(N)$ \\
\hline 3 & 1 & 7 & 4 & 6 \\
\hline 5 & 6 & 23 & 16 & 20 \\
\hline 7 & 13 & 48 & 36 & 42 \\
\hline
\end{tabular}

(c) FBPT of length 7 :

$$
\begin{aligned}
y(i) & =X(i)-X(6), i=1,2,3,4,5 \\
Y(1) & =Z^{4} y(1) \\
Y(2) & =Z^{2} y(2) \\
Y(3) & =Z^{4} Y(2) \\
Y(4) & =Z^{6} y(3) \\
Y(5) & =Z^{2} y(4) \\
Y(6) & =Z^{4} Y(5) \\
Y(7) & =Z^{3} y(5) \\
Y(8) & =Z^{2} Y(7) \\
Y(9) & =Z^{4}(y(4)+Y(1)+Y(4)) \\
Y(10) & =Z^{2}(y(1)+Y(2)+Y(6)) \\
Y(11) & =Z^{3}(y(1)+Y(4)+Y(5)+Y(8)) \\
Y(12) & =Z^{6}(y(5)+Y(2)+Y(4)) \\
Y(13) & =Z(y(3)+Y(1)+Y(2)+Y(7)) \\
H(0) & =\sum_{i=1}^{6} X(i) \\
H(1) & =-X(6)+Y(2)+Y(8)+Y(9) \\
H(2) & =-X(6)+Y(4)+Y(7)+Y(10) \\
H(3) & =-X(6)+Y(3)+Y(11) \\
H(4) & =-X(6)+Y(1)+Y(5)+Y(12) \\
H(5) & =-X(6)+Y(6)+Y(13) \\
\bar{X}(i) & =X(0)+H(i), i=0,1,2,3,4,5 \\
\bar{X}(6) & =X(0)-\sum_{i=0}^{5} H(i) \\
Y & \\
Y &
\end{aligned}
$$

\section{Performance comparison}

It is clear, from eqn. 1, that the direct computation of an $N$-point FBPT needs $(N-1)^{2}$ multiplications by powers of $Z$ (digit shifts) and $N(N-1)$ polynomial additions. Table 1 shows the performance comparison between the proposed algorithm and the direct approach, where $M(N)$ Table 1

and $A(N)$ stand for the number of digit shifts and the number of polynomial additions required for an $N$-point FBPT, respectively.

From Table 1, we conclude that the proposed algorithm is more efficient than the direct approach when the ratio of the cost of a polynomial addition to that of the shifting operation is less than 3.33 .

As pointed out by the referees, this condition could be verified on the basis of speed or gate count. The speed superiority of shifting to addition is obvious, so in the following discussions the cost of an operation will be defined in terms of gate count. To do this we make the following simple assumptions regarding the arithmetic functions used in References 10 and 11 :

(i) An adder for an input word length of $m$ bits has a complexity of $16 \mathrm{~m}$ logic gates, i.e.

$$
G_{a d d}=16 \mathrm{~m}
$$

(ii) A parallel access register has a complexity of $5 m$ logic gates for an $m$-bit word, i.e.

$$
G_{r e g}=5 m
$$

Then, for the polynomials of $m$-bit coefficient precision and degree $N$, the ratio of the cost (the number of gate count) of polynomial addition to the cost of shifting can be represented as

$$
\frac{N G_{a d d}}{N G_{\text {reg }}}=\frac{16}{5}=3.2<3.33
$$

This completes our verification.

4 References

1 NUSSBAUMER, H.J.: 'Fast fourier transform and convolution algorithms' (Springer-Verlag, 1981)

2 NÜSSBAUMER, H.J.: 'Digital filtering using forms', Electron. Lett., 1977, 13, (13), pp. 386-387

3 MCCLELLAN, J.H., and RADAR, C.M.: 'Number theory in digital signal processing' (Prentice-Hall Inc., 1979)

4 ARAMBERPOLA, B., and RAYNER, P.J.W.: 'Discrete transforms over polynomial rings with applications in computing multidimensional convolutions', IEEE Trans., 1980 ASSP-28, pp. 407-414

WU, J.-L., and PEI, S.-C.: 'Fast biased polynomial transforms', IEEE Trans., 1986, ASSP-34, pp. 383-385

6 PEI, S.-C., and WU, J.-L.: 'Fast biased polynomial transforms for 2D convolutions', Electron. Lett., 1981, 17, (15), pp. 547-548

WU, J.-L., and PEI, S.C.. 'Fast biased polynomial transforms for 2D prime length discrete Fourier transforms', Electron. Lett., 1984, PEI S.C and WU,

WU, J-L : 'Pipeline fast biased polynimial transform architecture for 2D convolutions', IEEE Int. Conf. Commun., May 14-17 1984, pp. 238-241

DUHAMEL $\mathrm{P}$ and HOLLMANN, $\mathrm{H}$. On discrete Fourier tansform (number theortic transorms) form (number-theoretic transforms) with minimum number of rota-
tions (shifts)', Signal Process., 1988, 14, pp. 237-241 WARD, J.S., BARTON, P., ROBERTS, G., and STANIER, W. 'Figures of merit for VLSI implementations of digital signal pro'Figures of merit for VLSI implementations of digital signal pro-
cessing algorithms', IEE Proc., F., 1984, 131, (1), pp. 64-70 1 PFRERA W A : 'A form hardware implementation in VLSI', IEEE Trans, 1987, ASSP35, pp. $1750-1760$

IEE PROCEEDINGS, Vol. 137, Pt. E, No. 2, MARCH 1990 